\title{
Identification of Patients with New-Onset Heart Failure and Reduced Ejection Fraction in Danish Administrative Registers
}

This article was published in the following Dove Press journal:

Clinical Epidemiology

\author{
Christian Madelaire ${ }^{1}$ \\ Finn Gustafsson ${ }^{2,3}$ \\ Lars Køber $\mathbb{D}^{2}$ \\ Christian Torp-Pedersen ${ }^{4,5}$ \\ Charlotte Andersson 1,6 \\ Søren Lund Kristensen ${ }^{2}$ \\ Gunnar Gislason (D) ${ }^{1,7}$ \\ Morten Schou' \\ 'Department of Cardiology, Herlev and \\ Gentofte University Hospital, \\ Copenhagen, Denmark; ${ }^{2}$ Department of \\ Cardiology, Rigshospitalet, University of \\ Copenhagen, Copenhagen, Denmark; \\ ${ }^{3}$ Department of Clinical Medicine, \\ University of Copenhagen, Copenhagen, \\ Denmark; ${ }^{4}$ Department of Cardiology \\ and Clinical Research, Nordsjaellands \\ Hospital, Hilleroed, Denmark; \\ ${ }^{5}$ Department of Cardiology, Aalborg \\ University Hospital, Aalborg, Denmark; \\ ${ }^{6}$ Department of Medicine, Section of \\ Cardiovascular Medicine, Boston Medical \\ Center, Boston, MA, USA; ${ }^{7}$ Danish Heart \\ Foundation, Copenhagen, Denmark
}

Correspondence: Christian Madelaire Department of Cardiology, Copenhagen University Hospital Gentofte,

Kildegårdsvej 28, Opgang 6, 3rd floor,

Copenhagen, Denmark

Tel +45 20928645

Email cras0185@regionh.dk
Background: In Danish administrative registers, ejection fraction (EF) is not recorded, which is a considerable limitation for correct subclassification of patients with heart failure (HF). We hypothesized that a diagnosis of HF combined with the recorded prescription of both renin-angiotensin system (RAS) inhibitors and beta- blockers $(R A S i+B B)$ within 120 days could identify patients with HF and reduced ejection fraction ( $\mathrm{EF} \leq 40 \%$ ) (HFrEF).

Methods: On two sites, we identified all patients with a first-time registration of $\mathrm{HF}$ as primary hospital discharge diagnosis (ICD-10: I50) between June 1, 2016, and May 31, 2018 in inpatient or outpatient settings. Patients were included if they survived the initial 120 days after discharge. Reviewing patient records, we identified patients with $\mathrm{HFrEF}$, based on $\mathrm{EF} \leq$ $40 \%$ and reported HF symptoms. We registered the use of $R A S i+B B$ at 120 days and calculated sensitivity, specificity and predictive values.

Results: A total of 704 consecutive patients with a primary diagnosis of HF were included, of whom 541 (77\%) fulfilled the HFrEF criteria. Patients with HFrEF confirmed from patient records were younger (median age 73 compared to 79 years) and less frequently women (31\% compared to $56 \%$ ) compared to non-HFrEF patients. At baseline, $24(4 \%)$ of $\mathrm{HFrEF}$ patients were treated with $R A S i+B B$ compared to $22(14 \%)$ of non-HFrEF patients. At 120 days, $460(85 \%)$ of HFrEF patients received $R A S i+B B$ as compared to $25(15 \%)$ of nonHFrEF patients. This resulted in a positive predictive value of $95 \%$, sensitivity of $85 \%$ and specificity of $85 \%$.

Conclusion: In Denmark, the ICD-10 HF diagnosis combined with recorded $R A S i+B B$ treatment by 120 days after discharge has high positive predictive value and can accurately be used to identify patients with HFrEF.

Keywords: heart failure, reduced ejection fraction, register data, validation

\section{Introduction}

Danish administrative registers have a high level of consistency and completeness of data, and are, therefore, widely used for epidemiological studies. Due to a unique personal identification number given to all Danish citizens at birth or immigration, individuals can be identified in different registers and across different geographical regions within Denmark. ${ }^{1,2}$ Identification of patients with heart failure (HF), based on international classification of disease (ICD-10) codes has previously been evaluated in the Danish registers documenting a relatively high positive predictive value (PPV) ranging from $81 \%$ to $100 \%$ depending on which method was used for validation. $^{3-5}$ However, the ICD-10 system does not distinguish between HF with 
reduced (HFrEF) and preserved (HFpEF) ejection fraction (EF), as the ICD-10 code for HF (I50) comprises both.

The network of Danish heart failure clinics ensures that the majority of Danish citizens who are diagnosed with HFrEF are offered consultations in a dedicated HF clinic with a focus on uptitration of guideline-directed medical therapy and patient education in self-care, usually over a period of 4 to 6 months. ${ }^{6,7}$ Since renin-angiotensin system inhibitors (RASi) and beta-blockers (BB) are recommended for all patients with HFrEF, if tolerated, ${ }^{8}$ we hypothesized that patients with HFrEF who survived the initial 120 days ( $\sim$ months) after receiving the diagnosis, could be identified based on the ICD-10 discharge code and filled prescriptions of $R A S i+B B$ within that time period.

\section{Methods}

This study was a part of a quality assurance project, with the purpose of evaluating the proportion of patients with HF alive after 120 days, who had initiated RASi and/or BB within 90 and 120 days, respectively. In the National Patient Register, we identified all patients who received a first-time HF diagnosis (ICD-10: I50) as a primary discharge diagnosis on two sites, Gentofte University Hospital and Herlev University Hospital, within a two-year period from June 1, 2016 through May 31, 2018. Patients who survived the initial 120 days after the time of diagnosis were included. Based on the review of patient records, patients were considered to have HFrEF if symptoms of HF were reported and an $\mathrm{EF} \leq 40 \%$ was documented in the chart. If several echocardiograms had been performed, either during the hospitalization or in the outpatient clinic, the first available measurement of EF was used to define HFrEF status in accordance with clinical practice. Symptoms of HF were shortness of breath, reduced exercise tolerance, fatigue and/ or ankle swelling. Use of RASi (including angiotensinconverting enzyme inhibitors [ACEi] and angiotensin receptor blockers $[\mathrm{ARB}]$ ) and $\mathrm{BB}$ was assessed at baseline (treatment initiated before the time of diagnosis) and at 120 days after diagnosis.

\section{Statistical Methods}

Baseline characteristics were presented as absolute numbers and proportions for categorical variables and medians and inter-quartile ranges (IQR) for continuous variables. Variables with missing data were presented as proportions and IQR relative to the group of patients with available data. Based on the ICD-10 discharge coding diagnosis for heart failure (I50) and status of $R A S i+B B$ at 120 days, we calculated sensitivity, specificity and predictive values for HFrEF.

\section{Ethical Considerations}

Register studies and patient record review studies do not require ethical approval in Denmark. The present quality assurance project was approved by the hospital direction (workzone number: 19,000,557). Patient data was anonymized immediately, and individuals included in the analyses could not be identified at a later point.

\section{Results}

\section{Study Population}

A total of 704 patients had a first-time HF diagnosis between June 1, 2016 and May 31, 2018 and were still alive after 120 days. Of those, 541 (76.8\%) had welldescribed HFrEF. Less information was available for the non-HFrEF group $(n=163)$ and for $43(26.4 \%)$ patients an echocardiography had not been performed. Of the patients with HFrEF, 503 (93.0\%) attended the HF clinic compared to only $21(12.9 \%)$ of the non-HFrEF patients. Baseline characteristics are listed in Table 1. Comorbidity data was missing for 101 individuals - none of whom attended the HF clinic.

\section{Use of RASi and BB}

At baseline, 24 (4.4\%) patients with HFrEF and 22 (13.5\%) non-HFrEF patients were treated with $R A S i+B B$. After 120 days, $460(85.0 \%)$ patients with HFrEF and 25 (15.3\%) non-HFrEF patients were taking $R A S i+B B$. For patients with HFrEF who attended a HF clinic, 444 (88.3\%) were receiving $R A S i+B B$ therapy after 120 days, as illustrated in Table 2. Identifying patients with HFrEF among patients discharged with an 150 diagnosis, and based on treatment with $R A S i+B B$ was associated with a PPV of $94.8 \%$ as shown in Figure 1 and Table 3. This, however, came with a sensitivity of $85.0 \%$. An even higher PPV of $99.1 \%$ could be obtained by selecting patients who were naïve to either RASi or BB at the time of diagnosis. In this case, the sensitivity was $80.1 \%$.

\section{Patients with HFrEF and Without RASi and $B B$}

According to patient records, reasons for not initiating RASi and/or BB therapy in patients with HFrEF were most often low blood pressure, low heart rate (BB), or reduced renal function (RASi). However, comparing 
Table I Baseline Characteristics. Count/Median (Percentage, Inter-Quartile Range) [Data Available in \% Individuals]

\begin{tabular}{|l|l|l|}
\hline & HFrEF (n=54I) & Non-HFrEF (n=I63) \\
\hline Female sex & $169(31.2)[100.0]$ & $91(55.8)[100.0]$ \\
Age & $73.2(63.9,80.7)[100.0]$ & $79.2(71.8,85.5)[100.0]$ \\
Hospitalization with HF symptoms & $421(77.8)[100.0]$ & $83(50.9)[97.5]$ \\
Attending heart failure clinic & $503(93.0)[100.0]$ & $21(12.9)[100.0]$ \\
Ischemic heart disease & $167(30.9)[100.0]$ & $16(25.8)[38.0]$ \\
Diabetes & $81(15.2)[100.0]$ & $16(25.8)[38.0]$ \\
Atrial fibrillation/flutter & $224(41.4)[100.0]$ & $31(50.0)[38.0]$ \\
Claudication & $12(2.2)[100.0]$ & $<3(<4.8)[38.0]$ \\
Stroke & $52(9.6)[100.0]$ & $9(14.5)[38.0]$ \\
Chronic obstructive pulmonary disease & $70(12.9)[100.0]$ & $12(19.4)[38.0]$ \\
\hline Medical therapy & & $53(32.5 \%)[100.0]$ \\
$\quad$ Renin angiotensin system inhibitors & $108(20.0 \%)[100.0]$ & $56(34.4 \%)[100.0]$ \\
Beta-blocker & $85(15.7 \%)[100.0]$ & $22(13.5 \%)[100.0]$ \\
$\quad$ Renin angiotensin system inhibitors and beta-blocker & $24(4.4 \%)[100.0]$ & \\
\hline Clinical measurements & & $55(47,60)[73.6]$ \\
Left ventricular ejection fraction & $30(20,37)[100.0]$ & $10(25.0 \%)[24.5]$ \\
Increased level of creatinine & $99(19.3 \%)[95.0]$ & $<3(2.9 \%)[21.5]$ \\
Systolic blood pressure <100mmHg & $18(3.5 \%)[94.6]$ & $3(9.4 \%)[19.6]$ \\
Heart rate <60 beats per minute & $56(11.3 \%)[91.3]$ & $1375(224,3483)[13.5]$ \\
NTproBNP & $1510(619,3460)[79.7]$ & \\
\hline
\end{tabular}

Abbreviations: HFrEF, heart failure with reduced ejection fraction; HF, heart failure.

Table 2 Frequency of RASi and Beta-Blocker Therapy After HF Diagnosis

\begin{tabular}{|l|l|l|}
\hline & Baseline & I 20 Days \\
\hline HFrEF (n=54I) & & \\
RASi & $108(20.0)$ & $496(9 I .7)$ \\
Beta-blocker & $85(15.7)$ & $492(90.9)$ \\
RASi + beta-blocker & $24(4.4)$ & $460(85.0)$ \\
Neither & $372(68.8)$ & $13(2.4)$ \\
\hline HFrEF in HF clinic $(\mathbf{n = 5 0 3 )}$ & & \\
RASi & $94(18.7)$ & $473(94.0)$ \\
Beta-blocker & $69(13.7)$ & $469(93.2)$ \\
RASi + beta-blocker & $19(3.8)$ & $444(88.3)$ \\
Neither & $359(71.4)$ & $5(1.2)$ \\
\hline Non-HFrEF (n=I63) & & \\
RASi & $53(32.5)$ & $59(36.2)$ \\
Beta-blocker & $56(34.4)$ & $63(38.7)$ \\
RASi + beta-blocker & $22(13.5)$ & $25(15.3)$ \\
Neither & $76(46.6)$ & $66(40.5)$ \\
\hline Non-HFrEF in HF clinic $(\mathbf{n = 2}$ I) & & \\
RASi & $8(38.1)$ & $9(42.9)$ \\
Beta-blocker & $<3(<7.0)$ & $3(7.0)$ \\
RASi + beta-blocker & $<3(<7.0)$ & $<3(<7.0)$ \\
Neither & $1 \mathrm{I}(52.4)$ & $10(47.6)$ \\
\hline
\end{tabular}

baseline characteristics of patients with and without $R A S i$ $+B B$ at day 120 revealed no significant differences in presence on these three parameters. Patients without $R A S i+B B$ were older with a median age of $79.7(71.4,87.0)$ years.

\section{Patients Without Echocardiography}

Patients without a documented echocardiography, $n=43$, were classified as non-HFrEF. Among these patients, 6 (14\%) were taking $R A S I+B B$ at 120 days - the same 6 patients were treated with $R A S i+B B$ at baseline.

\section{Discussion}

This study demonstrates that a population of patients with HFrEF can be identified from the Danish registers based on diagnosis and medical prescriptions with a PPV of $95 \%$. The ability to accurately identify patients with HFrEF is important for the design and validity of future epidemiological studies.

To our knowledge, this study is the first to evaluate the identification of a specific HF subgroup in the Danish administrative registers. The PPV of the HF diagnosis has previously been evaluated with different approaches. 


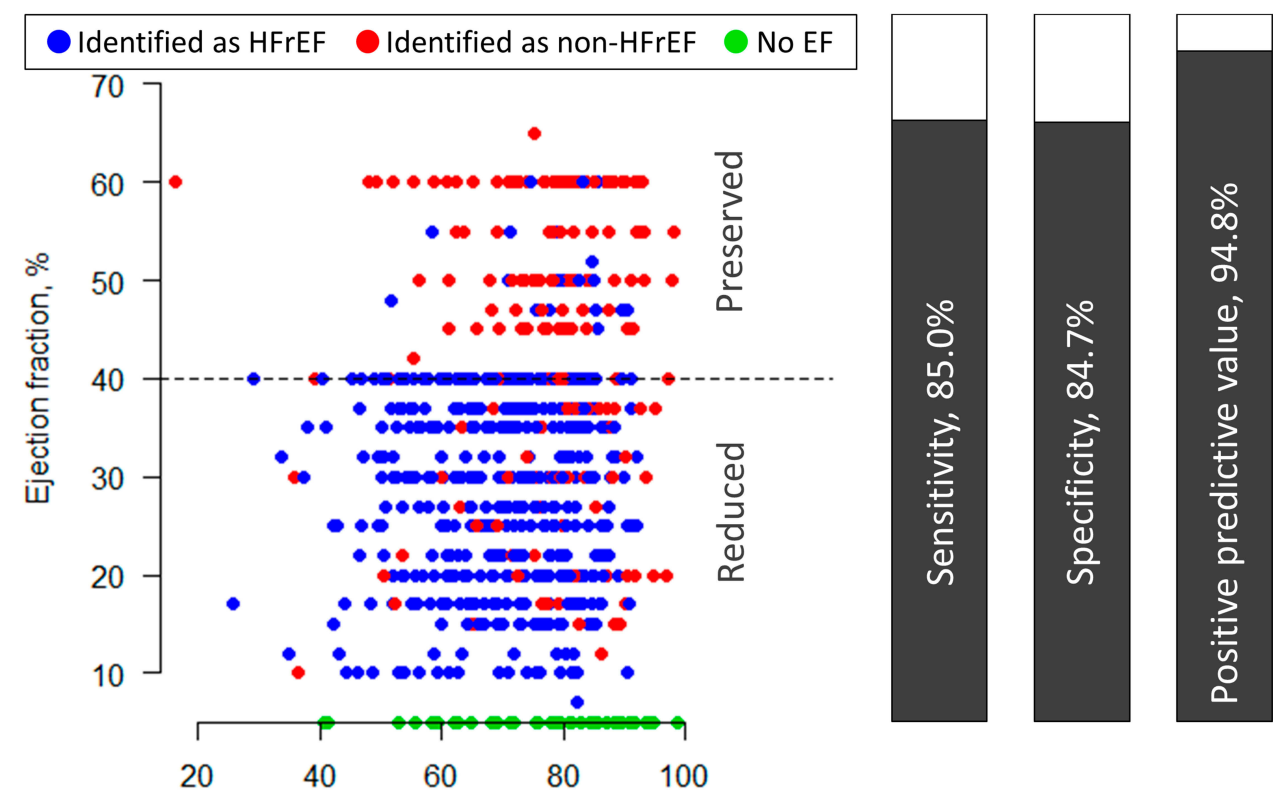

Age, years

Figure I Left side: Patients identified as HFrEF (blue) and non-HFrEF (red) plotted with age and EF. Patients without information on EF (green) plotted along the age-axis. Right side: Sensitivity, specificity and positive predictive value of confirmed HFrEF by using diagnosis code and RASi+BB at I20 days.

Abbreviations: $\mathrm{EF}$, ejection fraction; HFrEF, heart failure with reduced ejection fraction; RASi, renin-angiotensin-system inhibitor; $B B$, beta-blocker.

In a study using discharge summaries to verify the diagnosis, the PPV was found to be $100 \%$ for 50 patients reviewed. ${ }^{5}$ This method solely relied on the opinion of the discharging physician and it is unknown whether diagnostic tests were performed. This is a valuable method to ensure that the National Patients Register is reflecting the diagnoses given at discharge, but it is less valuable for determining whether the diagnostic criteria were met in the clinical situation. Therefore, important additional data were obtained, when 3201 hospitalized patients were evaluated with a clinical examination and echocardiography in another previous study. In this study, the PPV was found to be $81 \%$, as 30 patients out of 156 with a registered diagnosis, did not have HF according to the European Society

Table 3 Sensitivity, Specificity and Predictive Values of Confirmed HFrEF by Using Diagnosis Code and RASi+BB at 120 Days

\begin{tabular}{|l|l|l|l|}
\hline & Non-HFrEF & HFrEF & Total \\
\hline Not RASi and beta-blocker & 138 & 81 & 219 \\
RASi and beta-blocker & 25 & 460 & 485 \\
\hline Total & 163 & 541 & 704 \\
\hline
\end{tabular}

Notes: Sensitivity, $460 / 54 \mid=85.0 \%$. Specificity, I38/I63 $=84.7 \%$. Positive predictive value: $460 / 485=94.8 \%$. Negative predictive value: $138 / 219=63.0 \%$. of Cardiology criteria. ${ }^{3}$ Moreover, this study revealed a low sensitivity of $29 \%$ (39\% for HFrEF) among all patients hospitalized. The study included patients admitted to any department and did not consider prior diagnoses, which may explain the low sensitivity, as the HF diagnosis patient with well-known and well-treated HF may not have been recorded if the patient was admitted for non-cardiovascular comorbidity. The sensitivity of the register-based approach documented in the present study is not comparable to the study by Kumler, as the current study is derived from a population with registered HF. Consequently, the results of the current study reflect the proportion of true HFrEF among patients with a HF diagnosis identified using our definition. The main purpose, however, was to estimate the PPV of HFrEF, and we found that it was surprisingly high even when only using the diagnosis $(541 / 704)=77 \%$. When adding $\mathrm{RASi}+\mathrm{BB}$ use to the definition, 219 patients were redefined as non-HFrEF, of whom, at least 81 had HFrEF. In the group of non-HFrEF patients, the description of symptoms of HF in the medical records was less detailed, and NTproBNP was only measured in a few patients. Hence, it was, therefore, difficult to determine the proportion of true HFpEF. Due to a lack of echocardiography for some patients, we cannot rule out that they had HFrEF. If this were the case, for all these 
patients it would slightly increase the PPV to $96 \%$, while reducing the sensitivity to $80 \%$.

The proportion of HFpEF was low, even if all patients categorized as non-HFrEF had true HFpEF. This proportion has varied in the literature depending on definition, method and clinical settings. From the European Society of Cardiology HF Long-Term Registry, it has been reported that a third of the patients with chronic HF had an EF $>40 \% .{ }^{9}$ In the hospital, HFpEF may be underreported in the presence of comorbidity, and it is unknown how many patients are merely treated symptomatically with diuretic therapy without having a registered HF diagnosis. In a recent Danish study examining patients with risk factors for $\mathrm{HF}$ but without known $\mathrm{HF}, 37 \%$ had an abnormal echocardiography but no symptoms of HF (stage B HF), while $18 \%$ had abnormal echocardiography and symptoms of HF (stage C HF). ${ }^{10}$ Among those with unrecognized HF (stage C) $94 \%$ had an $\mathrm{EF}>40 \%$, which supports the hypothesis of HFpEF being underdiagnosed. Further, even recognized HFpEF may have low registration rates in Denmark due to our public tax-paid health care system, where registration of a diagnosis is not associated with the same financial or competitive benefits for the hospitals as in other countries. This does not affect the validity of the data presented, but it may affect the generalizability of the proposed method to other countries; the PPV will be lower in populations with a higher proportion of HFpEF. Therefore, this method should be tested in other cohorts before being applied in settings different from the Danish.

A considerable part of the cohort did not receive $R A S i$ $+B B$ by 120 days. The reported rationale of avoiding/ postponing initiation of RASi was often a high level of creatinine, while BB was often avoided/postponed due to low blood pressure or low heart rate. However, we observed no significant difference in the frequency of low blood pressure, heart rate, or high level of creatinine at baseline between patients with and without $R A S i+B B$ at day 120 . It is likely that the treatment decision is partly based on the patients' clinical appearance, and parameters such as a low cardiac output state may indicate full uptitration of RASi before initiation of (and therefore postponing) BB. Another explanation could be that patients who experienced an early readmission had a delay in otherwise planned interventions and up-titration of medical therapy. However, early readmission rates are low in Denmark, so this should only apply to a minority of the patients. ${ }^{11}$ Noticeably, only $2 \%$ of the patients with HFrEF initiated neither RASi nor BB. Further, it is unknown how many of these patients initiated $R A S i+B B$ at a later point after 120 days.

\section{Strengths and Limitations}

The Danish health care system is highly homogenous in terms of diagnostic approaches, treatment strategies and quality of care, which is a major strength for a study as the present, relying on nationwide generalizability. The two hospitals included were both university hospitals, but represented both invasive and non-invasive cardiology departments.

Defining HFrEF in the Danish registers using the I50 code and post-discharge initiation of $R A S i+B B$ within a certain period inherently results in the exclusion of patients dying within this period, which introduces selection bias towards the younger and less frail patients. Unfortunately, we did not have data regarding these excluded patients; however, in previous work on data from the nationwide registers, $20 \%$ of 172,580 patients diagnosed with HF from 2000 to 2012 died within 120 days - including those who died during the initial hospitalization. ${ }^{12}$ The distribution of early death between patients with and without HFrEF is unknown. Further, the present analyses showed a loss of $15 \%$ of the patients with HFrEF surviving the initial 120 days, using the proposed method. These patients were older than the included patients were and, likely, more frail. This should be kept in mind when applying the method in future epidemiological research, but it may be less important for studies concerning patients with chronic HF.

We acknowledge that estimating EF is susceptible to inter- and intra-observer variability and patients with an EF close to $40 \%$ could be misclassified. However, this reflects the daily clinical practice and is not considered a limitation for the purpose of this study.

\section{Conclusions}

In Danish administrative registers, the combination of a HF and initiation of RASi as well as BB by 120 days after diagnosis defines HFrEF with a PPV of $95 \%$. Despite a sensitivity of $85 \%$, using this method for register studies is reasonable.

\section{Funding}

This work was supported by the Danish Heart Foundation, Copenhagen, Denmark [grant number 17-R116-A761022048]. 


\section{Disclosure}

Dr Christian Madelaire reports grants from The Danish Heart Foundation, during the conduct of the study. Professor Finn Gustafsson reports grants, personal fees from Abbott, personal fees from Novartis, Orion Pharma, Pfizer, Carmat, Boehringer-Ingelheim, Astra-Zeneca, outside the submitted work. Professor Lars Køber reports personal fees and Speakers honorarium from Novartis, AstraZeneca and Boehringer, outside the submitted work. Professor Christian Torp-Pedersen reports grants from Bayer and Novo Nordisk, outside the submitted work. The authors report no other conflicts of interest in this work.

\section{References}

1. Schmidt M, Schmidt SAJ, Sandegaard JL, Ehrenstein V, Pedersen L, Sørensen HT. The Danish national patient registry: a review of content, data quality, and research potential. Clin Epidemiol. 2015;7:449490. doi:10.2147/CLEP.S91125

2. Kildemoes HW, Sørensen HT, Hallas J. The Danish national prescription registry. Scand J Public Health. 2011;39(7 Suppl):38-41. doi: $10.1177 / 1403494810394717$

3. Kümler T, Gislason GH, Kirk V, et al. Accuracy of a heart failure diagnosis in administrative registers. Eur J Heart Fail. 2008;10 (7):658-660. doi:10.1016/j.ejheart.2008.05.006

4. Mard S, Nielsen FE. Positive predictive value and impact of misdiagnosis of a heart failure diagnosis in administrative registers among patients admitted to a University Hospital cardiac care unit. Clin Epidemiol. 2010;2:235-239. doi:10.2147/CLEP.S12457
5. Thygesen SK, Christiansen CF, Christensen S, Lash TL, Sørensen HT. The predictive value of ICD-10 diagnostic coding used to assess Charlson comorbidity index conditions in the population-based Danish national registry of patients. BMC Med Res Methodol. 2011;11:83. doi:10.1186/1471-2288-11-83

6. Gustafsson F, Ulriksen H, Villadsen H, Nielsen H, Andersen BB, Hildebrandt R. Prevalence and characteristics of heart failure clinics in Denmark-design of the Danish heart failure clinics network. Eur $J$ Heart Fail. 2005;7(2):283-284. doi:10.1016/j.ejheart.2004.03.016

7. Gustafsson F, Schou M, Videbaek L, et al. Treatment with betablockers in nurse-led heart failure clinics: titration efficacy and predictors of failure. Eur J Heart Fail. 2007;Sep(9):910-916. doi:10.1016/j.ejheart.2007.05.008

8. Ponikowski P, Voors AA, Anker SD, et al. 2016 ESC guidelines for the diagnosis and treatment of acute and chronic heart failure: the task force for the diagnosis and treatment of acute and chronic heart failure of the European Society of Cardiology (ESC). Developed with the special contribution of the Heart Failure Association (HFA) of the ESC. Eur J Heart Fail. 2016;18(8):891-975. doi:10.1002/ejhf.592

9. Crespo-Leiro MG, Anker SD, Maggioni AP, et al. European Society of Cardiology Heart Failure Long-Term Registry (ESC-HF-LT): 1year follow-up outcomes and differences across regions. Eur J Heart Fail. 2016;18(6):613-625. doi:10.1002/ejhf.566

10. Gaborit FS, Kistorp C, Kümler T, et al. Prevalence of early stages of heart failure in an elderly risk population: the Copenhagen heart failure risk study. Open Heart. 2019;6(1):e000840. doi:10.1136/ openhrt-2018-000840

11. Butt JH, Fosbøl EL, Gerds TA, et al. Readmission and death in patients admitted with new-onset versus worsening of chronic heart failure: insights from a nationwide cohort. Eur J Heart Fail. 2020. doi:10.1002/ejhf. 1800

12. Madelaire C, Gustafsson F, Stevenson LW, et al. Favorable five-year outcomes for heart failure diagnosed in younger patients without severe comorbidity. Int J Cardiol. 2020;305:106-112. doi:10.1016/j. ijcard.2020.01.055
Clinical Epidemiology

\section{Publish your work in this journal}

Clinical Epidemiology is an international, peer-reviewed, open access, online journal focusing on disease and drug epidemiology, identification of risk factors and screening procedures to develop optimal preventative initiatives and programs. Specific topics include: diagnosis, prognosis, treatment, screening, prevention, risk factor modification, systematic reviews, risk \& safety of medical interventions, epidemiology \& biostatistical methods, and evaluation of guidelines, translational medicine, health policies \& economic evaluations. The manuscript management system is completely online and includes a very quick and fair peer-review system, which is all easy to use.

\section{Dovepress}

